\title{
Effectiveness of morphological and spectral heartbeat characterization on arrhythmia clustering for Holter recordings
}

\author{
Cristian Castro-Hoyos ${ }^{a}$, Diego Hernán Peluffo-Ordoñez ${ }^{b}$, Jose Luis Rodríguez-Sotelo ${ }^{c}$ and \\ Germán Castellanos-Domínguez ${ }^{a}$ \\ ${ }^{a}$ Universidad Nacional de Colombia - Manizales \\ ${ }^{b}$ Universidad Cooperativa de Colombia - Pasto \\ ${ }^{c}$ Universidad Autónoma de Manizales
}

\begin{abstract}
Heartbeat characterization is an important issue in cardiac assistance diagnosis systems. In particular, wide sets of features are commonly used in long term electrocardiographic signals. Then, if such a feature space does not represent properly the arrhythmias to be grouped, classification or clustering process may fail. In this work a suitable feature set for different heartbeat types is studied, involving morphology, representation and time-frequency features. To determine what kind of features generate better clusters, feature selection procedure is used and assessed by means clustering validity measures. Then the feature subset is shown to produce fine clustering that yields into high sensitivity and specificity values for a broad range of heartbeat types.
\end{abstract}

Keywords Clustering methods, Feature extraction, Signal analysis

\section{INTRODUCTION}

As a tool for clinical settings and medical information management, the diagnosis of transient and infrequent cardiac arrhythmias is employed, usually carried out over long term electrocardiographic signals (Holter ECG) holding a huge amount of heartbeats. ${ }^{1}$ Since manual analysis and inspection of a whole Holter recording is a time consuming and exasperating task, then, heartbeat analysis should be provided through an automatic system. Among other important aspects to take into consideration, a proper heartbeat characterization must be ensured as a starting point for reducing the computational burden but achieving compact and consistent groups. Therefore, the effectiveness of extracted heartbeat feature set for heartbeat clustering should be strongly considered. This issue has been addressed before using techniques based on either improved clustering or feature selection/extraction, by instance in. ${ }^{2}$

Among the known baseline projection approaches, Principal Component Analysis (PCA) and the $Q-\alpha$ algorithm are recommended for feature selection analysis over a wide set of features for separation of heartbeats in long-term ECG signals, as discussed in. ${ }^{1}$ In both cases, the original representation space is projected onto a new space preserving the relevant information in terms of an explained variance criterion. However, they ignore the relationship matching the projected space with the original feature space that may supply additional information. Furthermore, a proper relevance analysis for feature selection taking advantage of this relationship might provide an insight on how the underlying data information is to be associated, for example, using different cluster separability and/or compactness measures, even more, identification of most suitable features for heartbeat clustering might be provided by considering validity measures.

Based on clustering measures, this work discusses the effectiveness of commonly used features for heartbeat clustering. To this end, we suggest linear combination of the leading eigenvectors of the covariance matrix as a relevance vector, pointing out the address of the features holding the most discriminating evidence. ${ }^{3}$ In particular, we consider two basic principles of feature generation: morphological (derived from heart rate variability (HRV) and prematurity) and spectralbased parameters. Extracted from the latter generation principle, besides conventional Fourier, Discrete Wavelet Transform (DWT), and Hermite coefficients, we also use some here introduced features, namely parameters of a cosine modulated Gaussian atom. Experimental testing is provided using the MIT-BIH Arrhythmia Database. A total set of 181 features is considered to be further selected, in terms of provided relevance analysis. The overall feature selection procedure requires

Corresponding Author: Cristian Castro-Hoyos. Signal Processing and Recognition Group, Km. 9, Vía al aeropuerto, Campus la Nubia, Caldas, Manizales, Colombia, Email: ccastroh@unal.edu.co, Telephone: (+576) 8879300 Ext. 55713 
manual setting of optimal parameters, to this end, validity clustering measures Adjusted Rand Index (ARI) ${ }^{4}$ and Normalized Mutual Information (NMI), ${ }^{5}$ are used as criteria for experimental tuning. And selected feature ensemble is assessed by means of sensitivity and specificity measures, so the underlying pathology discrimination can be further analyzed.

This paper is organized as follows: Section 2 outlines the methods for heartbeat characterization as well as for feature relevance analysis. In sections 3 and 4 the experimental setup is described and results are discussed, respectively. Finally, conclusions and final remarks are presented in Section 5.

\section{MATERIALS AND METHODS}

\subsection{Heartbeat Feature Generation}

Morphological features: Heartbeats are usually discriminated by means of morphology, since most pathologies exhibit time-domain changes that are not consistent with normal heartbeats. At first, HRV-based features are considered as a discriminating parameter, specifically to deal with premature ventricular heartbeats as they have high shape similarity with normal heartbeats, but differ in timing and phase of the main fiducial points. ${ }^{1}$ Thereby, three HRV-based features are considered,

$$
x_{1}=r_{j}-r_{j-1}, \quad x_{2}=r_{j-1}-r_{j-2}, \quad x_{3}=r_{j+1}-r_{j},
$$

where $r_{j}$ is the $j$-th R-peak time location, and $x_{1}$ is the $\mathrm{R}$ to $\mathrm{R}$ interval, $x_{2}$ is the previous $\mathrm{R}$ to $\mathrm{R}$ interval and $x_{3}$ the posterior $\mathrm{R}$ to $\mathrm{R}$ interval.

Heart rate changes are not only characterized by RR intervals, differences between consecutive heartbeats are also considered as features, as the RR interval information of RR has been already introduced above, only the Atrial $P$ wave morphology index, which is sensible to any increase in heart rate is used,

$$
x_{4}=\left(\frac{x_{3}}{x_{1}}\right)^{2}+\left(\frac{x_{2}}{x_{1}}\right)^{2}+\left(\frac{1}{3} \sum_{i=1}^{3} x_{i}^{2} \log \left(x_{i}\right)^{2}\right) .
$$

Lastly, the following subset of 3 morphological based features are used: $x_{5}$ the dynamic time warping (DTW) dissimilarity index, $x_{6}$ polarity of QRS complex and $x_{7}$ the energy of QRS complex. In addition the minimum to maximum ratio and variance of the QRS complex are added as features $x_{8}=\widetilde{q}$ and $x_{9}=\operatorname{var}\{\boldsymbol{q}\}$, where vector $\boldsymbol{q}$ is the QRS complex at hand, and $\widetilde{z}$ is defined as

$$
\widetilde{z}=\frac{\min (z)}{\max (z)}
$$

where $z$ is a time-series feature vector, such as wavelet coefficients and min and max are the minimum and maximum amplitude values of vector $z$, respectively.

Spectral-based Features: Even though morphological and time domain features are discriminating attributes of most heartbeats, some pathological heartbeats are not represented by the previous feature subset, spectral attributes such as Fourier coefficients and Hermite basis functions ${ }^{6}$ are used to give information derived from the frequency behavior of the signals. Namely, features $x_{10}, \ldots, x_{14}$ correspond to the first five fourier coefficients (ranging from 1 to $20 \mathrm{~Hz}$ ) and features $x_{15}, \ldots, x_{25}$ are the Hermite coefficients, estimated from the QRS complex spectra as recommended in. ${ }^{6}$

The DWT coefficients are considered regarding they provide with information about the spectral changes in different frequency bands of the ECG signal. ${ }^{7}$ In particular, the second order Daubechies wavelet $\left(D B_{2}\right)$ is used given its shape similarity with normal heartbeats. Most information of ECG signals is contained in low frequency bands, namely $0.1 \mathrm{~Hz}$ to $30 \mathrm{~Hz}$, hence an appropriate level for DWT must be chosen. The DWT is perfomed over fixed length QRS complexes so the number of coefficients is constant over all considered heartbeats. Thereby for the $D B_{2}$ wavelet, which has a central frequency of $f_{c}=0.66667 \mathrm{~Hz}$ the subset of features $\left\{x_{26}, \ldots, x_{164}\right\}$ is generated, and related with specific spectral bands as shown in Table 1.

In addition, the minimum to maximum ratio of each vector of coefficients is also calculated as features $x_{165}, \ldots, x_{170}=$ $\left\{\widetilde{A}_{5}, \widetilde{D}_{5}, \ldots, \widetilde{D}_{1}\right\}$ and variances $x_{171}, \ldots, x_{176}=\left\{\operatorname{var}\left\{A_{5}\right\}, \operatorname{var}\left\{D_{5}\right\}, \ldots, \operatorname{var}\left\{D_{1}\right\}\right\}$. Lastly, a cosine modulated gaussian atom reconstructing the ECG signal is also considered. In particular, derived from the QRS complex spectrogram the 
Table 1: Frequency Bands Distribution for DWT- $D B_{2}$

\begin{tabular}{c|c|c|c}
\hline Coefficients & Level & Range $(H z)$ & Features \\
\hline & 1 & $120-240$ & $x_{99}, \ldots, x_{164}$ \\
& 2 & $60-120$ & $x_{65}, \ldots, x_{98}$ \\
Detail & 3 & $30-60$ & $x_{48}, \ldots, x_{64}$ \\
& 4 & $15-30$ & $x_{38}, \ldots, x_{47}$ \\
& 5 & $7.5-15$ & $x_{32}, \ldots, x_{37}$ \\
\hline Approximation & 5 & $0-7,5$ & $x_{26}, \ldots, x_{31}$ \\
\hline \hline
\end{tabular}

following parameters are extracted: amplitude $x_{177}$, frequency $x_{178}$, phase $x_{179}$, delay $x_{180}$, and support or duration $x_{181}$, as suggested for the decomposition of the heart sound signal in. ${ }^{8}$ Therefore, a total set of $p=181$ features are considered to be further reduced in terms of relevance analysis.

Finally, Table 2 shows a brief summary of the considered subsets of features and their corresponding cardiological target,

Table 2: Features Summary and cardiological target

\begin{tabular}{|c|c|c|c|}
\hline Features & Descriptior & & Target \\
\hline$x_{1}$ to $x_{4}$ & RR intervals & \multirow[b]{2}{*}{ Morphological } & Premature Heartbeats \\
\hline$x_{5}$ to $x_{9}$ & QRS complex & & Abnormal QRS complexes: Extrasystole, block branchs. \\
\hline$x_{1} 0$ to $x_{1} 4$ & Fourier Coefficients & \multirow{4}{*}{ Spectral } & Right and left bundle branch blocks \\
\hline$x_{1} 5$ to $x_{2} 5$ & Hermite Basis Coefficients & & Premature Ventricular Contractions \\
\hline$x_{2} 6$ to $x_{1} 76$ & Wavelet & & \multirow[b]{2}{*}{ Paced and fusion beats } \\
\hline$x_{1} 77$ to $x_{1} 81$ & Cosine modulated Atom & & \\
\hline
\end{tabular}

\subsection{Relevance of Generated Heartbeat Features}

As discussed in, ${ }^{3}$ for a data-set $\boldsymbol{X} \in \mathbb{R}^{n \times p}$, where $n$ is the number of frequency bins and $p$ the number of time samples, it is possible to find a set of weight values, namely relevance vector, with the main purpose of enabling an effective data dimensionality reduction with a proper quantitative ranking. This can be accomplished in an PCA-similar approach using the least squares function, called MSE-based relevance.

For a feature matrix $\boldsymbol{X}$ the feature relevance vector can be computed by means of the SVD of its covariance matrix $\boldsymbol{C}_{\boldsymbol{X}}=\boldsymbol{X}^{\top} \boldsymbol{X}$, so let,

$$
C_{X}=V W V^{\top}
$$

where $\boldsymbol{V}=\left\{\boldsymbol{v}_{i}, i \in p\right\}$ is the eigenvector matrix and $\boldsymbol{W}=\left\{w_{1} \geq w_{2} \geq \ldots \geq w_{i} \geq w_{p}, i \in p\right\}$ is a diagonal eigenvalues matrix. The relevance vector $\boldsymbol{w}=\left\{w_{i}: i \in p\right\}$ is given by, ${ }^{3}$

$$
\boldsymbol{w}=\mathbf{E}\left\{v_{i}\left(\overline{\boldsymbol{v}}_{i} \circ \overline{\boldsymbol{v}}_{i}\right): \forall i=1 \ldots, p\right\}
$$

Notation $\mathbf{E}\{\cdot\}$ stands for expectation operator, which is assumed as simple average in this case.

Now, let us introduce a lower dimension parameter $0<\chi<1$, such that the following vector of indexes $\boldsymbol{h}$ is given,

$$
\boldsymbol{h}=\underset{i}{\arg }\left(w_{i}>\chi ; i=1, \ldots, p\right)
$$

dimensionality reduction can be achieved by selecting only those features, i.e. the $h$-th indexed features, with a normalized relevance value higher than a predefined (manually set) $\chi$.

Now it can be seen that while PCA aims to find the linear projection $\boldsymbol{Y}=\boldsymbol{X} \boldsymbol{V}$, where $\boldsymbol{Y} \in \mathbb{R}^{n \times \rho}$ and $\rho<p$, MSE relevance uses the eigenvalues of the covariance matrix to provide with a weight value to each feature holding the initial space. This allows to maintain the interpretability of the classification features instead of PCA where interpretability in lost in the projection. Now, this process becomes a feature selection methodology when a reduced number of eigenvectors and their respective eigenvalues is used. 


\section{EXPERIMENTAL SET-UP}

\subsection{ECG Database and preprocessing}

The data collection from MIT-BIH is provided with a wide range of heartbeat types in a set of 48 recordings, each one sampled at rate of $360 \mathrm{~Hz}$ and lasting 30 minutes long. ${ }^{9}$ A subset of 18 recordings with a variety of cardiac phenomena and both balanced and unbalanced classes was chosen, namely records: 104, 106, 107, 109, 114, 116, 119, 203, 205, 208, 210, $214,215,217,221,228,231,233$. Additionally the labels of each heartbeat are provided to the corresponding annotation code: Normal, Left-bundle branch block, Right bundle branch block, Ventricular contraction, Fusion of ventricular and normal, fusion of paced and normal and Paced. As regards the preprocessing stage, each recorded holter ECG signal, $s(t)$ is initially subject to discrete transformation, that is, $s=\left\{s_{n}\right\}$ where $n \triangleq s\left[n T_{s}\right]$, being $n \in \mathbb{N}$ and $T_{s}=1 / F_{s}$ the sampling period. Then, to prevent biasing the obtained ECG vector is normalized with the standard score (z-scores) approach, i.e.,

$$
\hat{\boldsymbol{s}}=\frac{\boldsymbol{s}-\mathbf{E}\{\boldsymbol{s}\}}{\operatorname{var}\{\boldsymbol{s}\}} .
$$

Afterwards, the unbiased vector $\hat{\boldsymbol{s}}$ is filtered to reduce interferences from power line and base line wander, according to the procedures given in ${ }^{10}$ and,${ }^{11}$ respectively.

\subsection{Effectiveness Estimation of Heartbeat Feature Set}

Each recording contains annotations of the $r$-peaks $\left(r_{j}\right.$ time locations, where $j=1, \ldots, n$ of all heartbeats in signal $\hat{\boldsymbol{s}}$. Feature extraction was performed over fixed length signal portions containing the QRS complex, that is, a rectangular window of length $300 \mathrm{~ms}$ centered at $r_{j}$. Hence a feature matrix $\boldsymbol{X} \in \mathbb{R}^{n \times p}$ where $p=181$ is the number of features distributed as explained in section 2.

Now for a given input feature matrix $\boldsymbol{X}$, it is necessary to identify the features that better discriminate the classes within, hence an heuristic search over the parameters $\rho$ and $\chi$ is performed. Now to each pair of values, a relevance vector is generated and consequently a subset of features. Finally, the reduced data-set $\widehat{\mathbf{X}} \in \mathbb{R}^{n \times \hat{p}}$ where $\hat{p}$ is the total of indexes contained in vector $\mathbf{h}$, i.e. the reduced number of features, is grouped by means of clustering algorithms and validity measures (ARI and NMI) are computed. This process is summarized in Algorithm 1

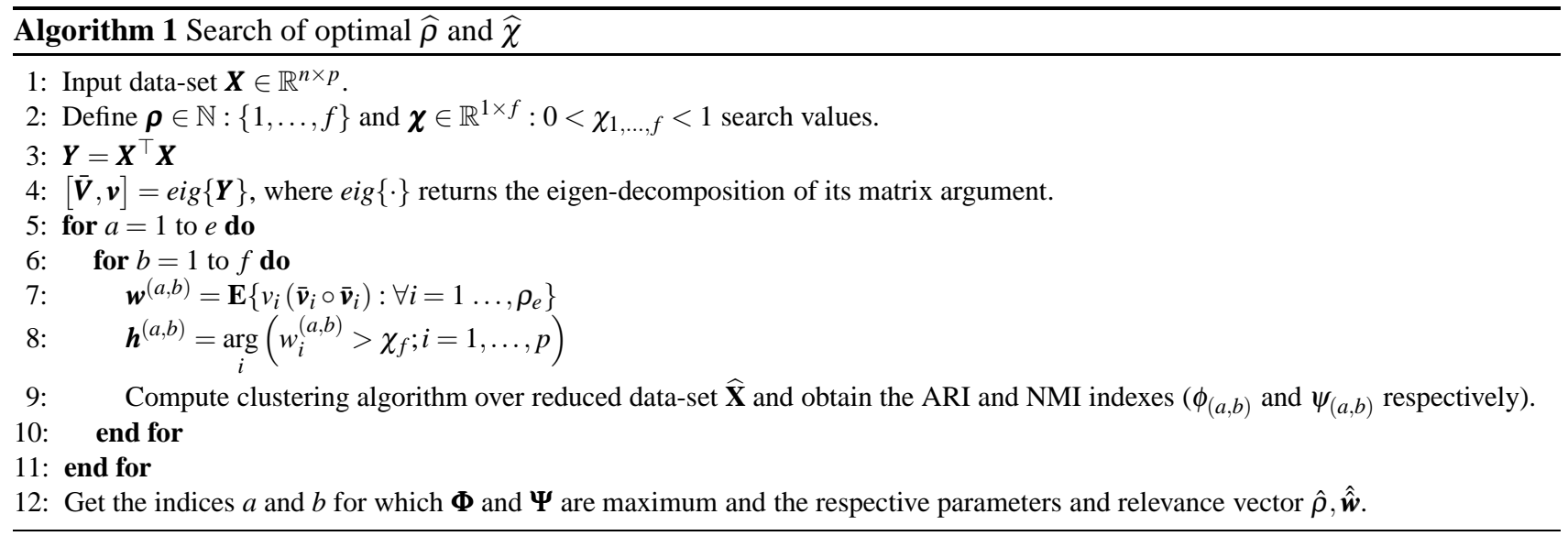

\subsection{Performed Accuracy of Reduced Heartbeat Feature Set}

In order to assess the performance of the feature selection, the tuning and experimental setting of $\rho$ and $\chi$ parameters is performed using Algorithm 1 with a kmeans clustering process (initial centroids provided by max-min algorithm) for each of the records obtaining a total of 18 relevance vectors. The reduced feature set is composed forming an average feature relevance from the whole database,

$$
\boldsymbol{w}_{\mu}=\mathbf{E}\left\{\hat{\boldsymbol{w}}_{i}: \forall i=1, \ldots, 18\right\}
$$


Now for the average relevance $\boldsymbol{w}_{\mu}$, features with relevance value higher than a given $\chi$ parameter $(0<\chi<1)$ are to be considered to form the final reduced ensemble of features, this parameters gives us the possibility to eliminate some other features that were not that constant over the analysis, that is, features that were relevant only once or twice, might not be necessary if a more robust clustering technique is to be used in the final clustering process. Finally to analyze the feature selection effectiveness, Sensitivity and Specificity ( $S e$ and $S p$ ) are computed for the results of kmeans clustering algorithm $(\mathrm{KM})^{12}$ over the final reduced data-sets.

\section{RESULTS AND DISCUSSION}

Initially, we set the search values $\boldsymbol{\rho}=\{3,4,7,11,15,19,23\}$ and $\boldsymbol{\chi}:\{0.82,0.85,0.88,0.91,0.94,0.97\}$ and kmeans clustering is used over the heuristic search defined in algorithm 1. Table 3 shows the results of the optimal found parameters over each data-set, additionally the resulting number of features $\bar{p}$ is also given.

Table 3: $\widehat{\rho}$ and $\widehat{\chi}$ results with respect to ARI and NMI.

\begin{tabular}{ccccccc}
\hline Rec & $\mathrm{K}$ & $\widehat{\rho}$ & $\widehat{\chi}$ & $\bar{p}$ & ARI & NMI \\
\hline 104 & 3 & 11 & 0.91 & 23 & 0.8246 & 0.7324 \\
106 & 2 & 7 & 0.82 & 62 & 0.9853 & 0.9603 \\
107 & 2 & 3 & 0.94 & 8 & 0.9421 & 0.8840 \\
109 & 2 & 7 & 0.91 & 28 & 0.8613 & 0.7507 \\
114 & 2 & 3 & 0.88 & 24 & 1.0000 & 1.0000 \\
116 & 2 & 7 & 0.94 & 28 & 0.9843 & 0.9545 \\
119 & 2 & 3 & 0.97 & 16 & 1.0000 & 1.0000 \\
203 & 2 & 23 & 0.88 & 62 & 0.8585 & 0.7238 \\
205 & 2 & 7 & 0.97 & 17 & 1.0000 & 1.0000 \\
208 & 3 & 3 & 0.82 & 36 & 0.9049 & 0.8369 \\
210 & 3 & 7 & 0.94 & 11 & 0.8418 & 0.7241 \\
214 & 2 & 7 & 0.94 & 17 & 0.7054 & 0.5980 \\
215 & 2 & 3 & 0.88 & 18 & 0.9638 & 0.9179 \\
217 & 4 & 7 & 0.97 & 7 & 0.9419 & 0.8384 \\
221 & 2 & 15 & 0.97 & 18 & 0.9812 & 0.9530 \\
228 & 2 & 3 & 0.97 & 9 & 0.7997 & 0.7024 \\
231 & 2 & 3 & 0.97 & 7 & 0.9970 & 0.9905 \\
233 & 2 & 19 & 0.85 & 123 & 0.7452 & 0.6663 \\
\hline \hline
\end{tabular}

Afterward, the average relevance vector is estimated in accordance to the procedure discussed in $\S 3.3$. Figure 1 shows the estimated average value of feature relevance over the whole ECG recording set. To make more clear the contribution, each considered heartbeat feature set is drawn with different color.

As seen, contrary to the assumption that the timing and morphology features $\left(x_{1}, \ldots, x_{9}\right)$ are better in discriminating the heartbeats classes, the set of considered spectral-based features are also competitive. Basically, some of derived from Fourier and DWT coefficients $\left(\left\{x_{10}, \ldots, x_{14}\right\}\right.$ and $\left\{x_{26}, \ldots, x_{164}\right\}$, respectively), in average, reach a high relevance.

Now, having conformed the average relevance, the $\widehat{\chi}$ parameter is set to $\widehat{\chi}=0.15$ and clustering is once again performed only with those features holding an average relevance value higher to $\widehat{\chi}$. It must be remarked though, that the parameter setting is not a trivial matter, and visual inspection of the average achieved relevance was required, where in Figure 1 its clearly visible that there is a remarked gap between the number of features with relevances higher to 0.1 and 0.2 thus the value was manually set in the midpoint. It can be seen that some morphological features reach high relevance, yet the subset is composed mostly of spectral-based features (fourier, hermite, DWT and atom parameters). Table 4 shows the sensibility and specificity results for the most relevant features. It can be seen that such features represent a wide range of heartbeat types. 


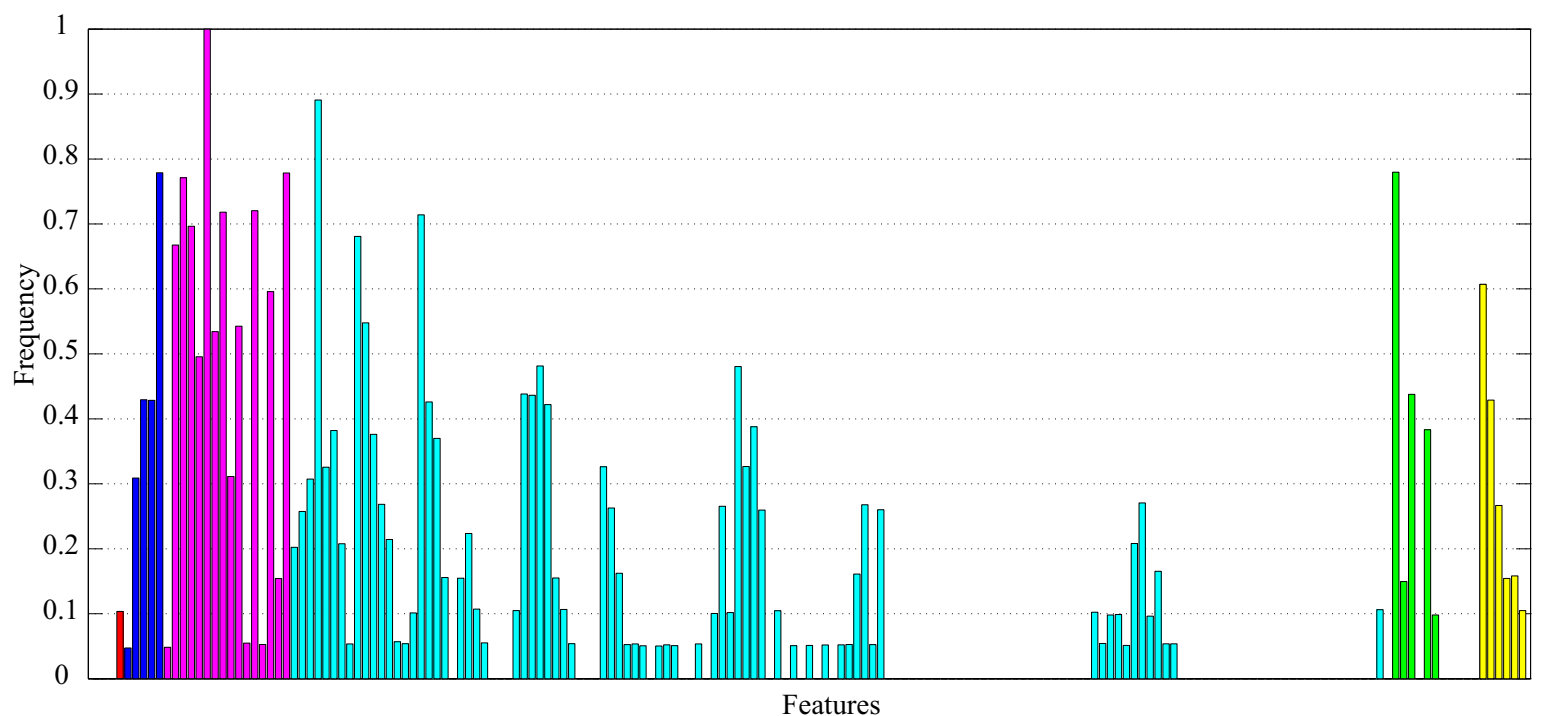

Figure 1: Estimated average value of feature relevance over the whole ECG recording set. $\left(\square-x_{1}, \ldots, x_{4}, \boldsymbol{\square}-x_{5}, \ldots, x_{9}\right.$, $\left.x_{10}, \ldots, x_{25}, \square-x_{26}, \ldots, x_{164}, \square-x_{165}, \ldots, x_{75}, \square-x_{176}, \ldots, x_{181}\right)$.

Table 4: Sensitivity and Specificity Results per record.

\begin{tabular}{|c|c|c|c|c|c|c|c|c|c|c|c|c|c|c|}
\hline Patient & $S e_{N}$ & $S p_{N}$ & $S e_{L}$ & $S p_{L}$ & $S e_{R}$ & $S p_{R}$ & $\mathrm{Se}_{V}$ & $S p_{V}$ & $S e_{F}$ & $S p_{F}$ & $S e_{f}$ & $S p_{f}$ & $S e_{P}$ & $S p_{P}$ \\
\hline 104 & 0.00 & 0.99 & & & & & & & & & 0.67 & 0.89 & 0.99 & 0.74 \\
\hline 106 & 0.99 & 0.99 & & & & & 0.99 & 0.99 & & & & & & \\
\hline 107 & & & & & & & 0.89 & 1.00 & & & & & 1.00 & 0.89 \\
\hline 109 & & & 0.99 & 0.28 & & & 0.28 & 0.99 & & & & & & \\
\hline 114 & 1.00 & 1.00 & & & & & 1.00 & 1.00 & & & & & & \\
\hline 116 & 0.99 & 0.99 & & & & & 0.99 & 0.99 & & & & & & \\
\hline 119 & 1.00 & 1.00 & & & & & 1.00 & 1.00 & & & & & & \\
\hline 203 & 0.98 & 0.91 & & & & & 0.91 & 0.98 & & & & & & \\
\hline 205 & 1.00 & 1.00 & & & & & 1.00 & 1.00 & & & & & & \\
\hline 208 & 1.00 & 0.95 & & & & & 0.94 & 0.99 & 0.82 & 0.98 & & & & \\
\hline 210 & 1.00 & 0.76 & & & & & 0.80 & 0.99 & & & & & & \\
\hline 214 & & & 1.00 & 0.62 & & & 0.62 & 1.00 & & & & & & \\
\hline 215 & 1.00 & 0.93 & & & & & 0.93 & 1.00 & & & & & & \\
\hline 217 & 1.00 & 0.91 & & & & & 0.85 & 1.00 & & & 0.38 & 0.98 & 0.99 & 1.00 \\
\hline 221 & 1.00 & 0.97 & & & & & 0.97 & 1.00 & & & & & & \\
\hline 228 & 1.00 & 0.77 & & & & & 0.77 & 1.00 & & & & & & \\
\hline 231 & 1.00 & 0.99 & & & 0.99 & 1.00 & & & & & & & & \\
\hline 233 & 1.00 & 0.65 & & & & & 0.65 & 1.00 & & & & & & \\
\hline
\end{tabular}

\section{CONCLUSIONS AND FUTURE WORK}

This works performs an study of some literature reported features for ECG arrhythmia characterization and uses a feature selection procedure to asses heartbeat clustering into high performance. The feature selection is done over heuristic search of the optimal parameters according to clustering validity measures ARI and NMI, taking advantage of the selection scheme it can be seen that spectral based features are more suitable to separate a broader spectrum of heartbeats, in contrast with the most common temporal and morphological features. Additionally as a result of feature selection, not only the clustering performance is enhanced but also the computational burden is reduced.

Although different in terms of nature, morphological and spectral based features are both important in heartbeat clustering. However the spectral-based features also include temporal information, as the DWT coefficients and Atom parameters, so they become more relevant in terms of clustering separability. This fact prompts to search for a mixed set of both features types that better represent each class of heartbeats. 
As future work, we attempt to carry out the evaluation of the extracted heartbeat feature sets by using a more complex optimization function allowing to include several clustering schemes exploring more elaborated relationships between projected and original feature spaces.

\section{Acknowledgments}

This work has been supported by the project "Discrimination of ECG Signals via Time-Frequency Representations" associated with the "Jóvenes Investigadores e Innovadores 2011" program by COLCIENCIAS, Regalías-Antioquia and the project "Servicio de Monitoreo Remoto de Actividad Cardíaca para el Tamizaje Clínico en la red de Telemedicina del Departamento de Caldas" financed by "Fondo Estampilla Universidad Nacional de Colombia Manizales - Universidad de Caldas".

As well, this work is partially supported by the project Sistema automatizado para la estimación del tamaño de lesión en el ventrículo izquierdo a causa de infarto de miocardio usando electrocardiografía, code: 249-028, by Grupo de Automática, at Universidad Autónoma de Manizales.

Authors would like to thank Faculty of Medicine and Faculty of Engineering from Universidad Cooperativa de Colombia - Pasto.

\section{REFERENCES}

1. J. Rodríguez-Sotelo, D. Peluffo-Ordoez, D. Cuesta-Frau, and G. Castellanos-Domínguez, "Unsupervised feature relevance analysis applied to improve ecg heartbeat clustering," Computer Methods and Programs in Biomedicine 108(1), pp. $250-261,2012$.

2. J. L. R. Sotelo, D. Peluffo, D. C. Frau, D. P. Ordnez, and G. C. Domínguez, "Non-parametric density-based clustering for cardiac arrhythmia analysis," Computers in cardiology. CINC, 2009.

3. A. Quiceno-Manrique, J. Alonso-Hernandez, C. Travieso-Gonzalez, M. Ferrer-Ballester, and G. CastellanosDominguez, "Detection of obstructive sleep apnea in ecg recordings using time-frequency distributions and dynamic features," in Engineering in Medicine and Biology Society, 2009. EMBC 2009. Annual International Conference of the IEEE, Engineering in Medicine and Biology Society, 2009. EMBC 2009. Annual International Conference of the IEEE, pp. $5559-5562$, sept. 2009.

4. L. Hubert and P. Arabie, “Comparing partitions,” Journal of Classification 1(2), pp. 193-218, 1985.

5. A. Strehl and J. Ghosh, "Cluster ensembles - a knowledge reuse framework for combining multiple partitions," Journal of Machine Learning Research 3, pp. 583-617, 2002.

6. M. Lagerholm, C. Peterson, G. Braccini, L. Edenbrandt, and L. Srnmo, "Clustering ecg complexes using hermite functions and self-organising maps," IEEE trans. on. Biomed. 48, pp. 838-847, 2000.

7. D. Cvetkovic, E. D. beyli, and I. Cosic, "Wavelet transform feature extraction from human ppg, ecg, and eeg signal responses to elf pemf exposures: A pilot study," Digital Signal Processing 18(5), pp. 861 - 874, 2008.

8. H. Tang, T. Li, T. Qiu, and Y. Park, "Segmentation of heart sounds based on dynamic clustering," Biomedical Signal Processing and Control 7(5), pp. 509 - 516, 2012.

9. G. Moody and M. R, “The impact of the mit-bih arrhythmia database," IEEE Transactions on engineering, medicine and biology 15(1), pp. 34-50, 1985.

10. S. Martens, M. Mischi, S. Oei, and J. Bergmans, "An improved adaptive power line interference canceller for electrocardiography," IEEE transactions on Biomedical Engineering 53, pp. 2220 - 2231, November 2006.

11. A. Roddy, "A filter to suppress ecg baseline wander and preserve st-segment accuracy in a real-time environment," Journal of Electrocardiology 24, pp. 315-323, October 1991.

12. A. Jain, M. Murty, and P. Flynn, "Data clustering: A review," ACM computer surveys 31, pp. 265-323, September 1999. 